\title{
Dynamic modeling of cis-regulatory circuits and gene expression prediction via cross-gene identification Li-Hsieh Lin ${ }^{\dagger 1}$, Hsiao-Ching Lee ${ }^{\dagger 2}$, Wen-Hsiung Li ${ }^{3,4}$ and Bor-Sen Chen*1
}

Address: ${ }^{1}$ Lab. of System Biology, National Tsing Hua University, 101, Sec 2, Kuang Fu Road, Hsinchu, 300, Taiwan., ${ }^{2}$ Department of Life Science \& Institute of Bioinformatics and Structural Biology, National Tsing Hua University, Hsinchu, 300, Taiwan., ${ }^{3}$ Department of Ecology and Evolution, University of Chicago, USA. and ${ }^{4}$ Genomics Research Center, Academia Sinica, Taipei, Taiwan.

Email: Li-Hsieh Lin - LHlin@moti.ee.nthu.edu.tw; Hsiao-Ching Lee - d884234@life.nthu.edu.tw; Wen-Hsiung Li - whli@uchicago.edu; BorSen Chen* - bschen@ee.nthu.edu.tw

* Corresponding author †Equal contributors

Published: 18 October 2005

BMC Bioinformatics 2005, 6:258 doi:10.1/86/147|-2105-6-258

Received: 19 March 2005

Accepted: 18 October 2005

This article is available from: http://www.biomedcentral.com//47/-2/05/6/258

(C) 2005 Lin et al; licensee BioMed Central Ltd.

This is an Open Access article distributed under the terms of the Creative Commons Attribution License (http://creativecommons.org/licenses/by/2.0), which permits unrestricted use, distribution, and reproduction in any medium, provided the original work is properly cited.

\begin{abstract}
Background: Gene expression programs depend on recognition of cis elements in promoter region of target genes by transcription factors (TFs), but how TFs regulate gene expression via recognition of cis elements is still not clear. To study this issue, we define the cis-regulatory circuit of a gene as a system that consists of its cis elements and the interactions among their recognizing TFs and develop a dynamic model to study the functional architecture and dynamics of the circuit. This is in contrast to traditional approaches where a cis-regulatory circuit is constructed by a mutagenesis or motif-deletion scheme. We estimate the regulatory functions of cis-regulatory circuits using microarray data.

Results: A novel cross-gene identification scheme is proposed to infer how multiple TFs coordinate to regulate gene transcription in the yeast cell cycle and to uncover hidden regulatory functions of a cis-regulatory circuit. Some advantages of this approach over most current methods are that it is based on data obtained from intact cis-regulatory circuits and that a dynamic model can quantitatively characterize the regulatory function of each TF and the interactions among the TFs. Our method may also be applicable to other genes if their expression profiles have been examined for a sufficiently long time.

Conclusion: In this study, we have developed a dynamic model to reconstruct cis-regulatory circuits and a cross-gene identification scheme to estimate the regulatory functions of the TFs that control the regulation of the genes under study. We have applied this method to cell cycle genes because the available expression profiles for these genes are long enough. Our method not only can quantify the regulatory strengths and synergy of the TFs but also can predict the expression profile of any gene having a subset of the cis elements studied.
\end{abstract}

\section{Background}

Cis-regulatory circuits have been applied to model cell cycle control and other developmental processes $[1,2]$. Recently, the genetic regulatory and transcriptional net- works of yeast have been studied [3-8]. However, in order to understand the regulation of any particular gene in the network, one should study carefully how the genes in the network collectively operate with remarkable precision in 
response to environmental cues and the structure and function of the cis-regulatory circuit of the gene [7]. The cis-regulatory circuit of a gene consists of its cis elements, i.e., binding motifs of transcription factor (TF), and the interactions among their recognizing TFs. The cis elements of a gene can be considered as the information processing units in the regulatory circuit; they receive multiple inputs from the TFs that bind the cis elements of the gene. The output is the instruction for the transcription apparatus to determine whether the gene is to be expressed at a specific rate or to be repressed $[9,10]$.

A cis-regulatory circuit may be regarded as a control device that is called into play by the TFs that have target sites inside the promoter [14-20]. A well-known example is the promoter region of the developmentally regulated endo 16 gene of the sea urchin [12-14]. It is about 2300 bp in length and consists of several clusters of target sites for distinct functions. Yuh et al. [12-14] have explored the function of each subregion of the endo 16 system and every target site within each subregion, using a cis-regulatory logic model.

However, a drawback of most current methods for inferring cis-regulatory circuits is that they rely on changing or deleting some binding site sequences (e.g., [12-14]), which may not provide intact functional information for reconstructing the cis-regulatory circuit. The deletion or mutation experiments may change or destroy the original cis-regulatory circuit structure. Using such data, one may lose significant interactions among transcription factors (TFs). Obviously, it is appealing to develop a method that can infer intact cis-regulatory circuits. Recently, there are some statistical and system level approaches to study the genome-wide transcription regulation and address cooperativity among TFs $([7-11,15,16])$. Important advances have been made toward understanding transcriptional regulatory networks. One strategy infers global networks directly from whole genome microarray data, and another strategy focuses on the identification of shared cis elements in the promoters of co-regulated genes, signified by similar expression profiles [8]. In this paper we develop a new method to combine microarray data and TF-binding location data by chromatin immunoprecipitation $[5,18]$ to study the regulatory and interaction functions of various cis elements with regard to the target gene. The data of Lee et al. 2002 [5] can reveal the in vivo physical interactions of TFs with their cis elements on the promoter and therefore can provide a more reliable view of functional interactions between TFs and cis elements. Combining these types of data and microarray data, we propose a novel cross-gene identification scheme to infer how multiple TFs coordinate to regulate gene transcription. Our approach is rather different from most existing statistical and system level methods for analyzing gene expression data. Our results show that this novel method is suitable for deciphering the complex TFs interactions and for predicting the gene expression. In addition, we also identify the dynamic regulatory functions of TFs interaction in the yeast cell cycle, which cannot be achieved by current methods.

\section{Results \\ Characterizing the cis-regulatory circuit of a gene}

In this study, there are two steps for characterizing the cisregulatory circuit of a gene. The first is to find a cluster of genes that includes the gene of interest and a number of other genes each of which shares a subset of cis elements with the gene of interest. Assuming a certain regulatory function for each of the TFs that recognize the cis elements of genes in the cluster and certain interaction functions among the TFs of a circuit, we set up dynamic equations for the cis-regulatory circuits of the genes in the cluster to describe their expression profiles. Since each gene in the cluster shares some cis elements with the gene of interest, a matrix of cis elements for the cluster of genes can be constructed. In this model, the regulatory functions of individual TFs and the interactions among TFs can be estimated from microarray data. In the second step, a cross-gene identification scheme is developed with an array of expression profiles of genes in the cluster (e.g., Spellman et al., 1998 [18]) to identify regulatory functions of the TFs and their possible interactions for each gene in the cluster; the parameters are estimated by the least square estimation algorithm.

Finally, plugging these estimated regulatory functions and interactions into the dynamic equations, one can explicitly describe the cis-regulatory circuit of the gene of interest.

\section{Choice of a cluster of genes}

As an illustration, suppose some genes of the yeast cell cycle are of interest. We find a cluster of genes for each gene of interest according to their cis elements found in Simon et al. 2001 [4]. To simplify the analysis, we consider only the nine TFs that are currently known to be important cell cycle TFs of the yeast (i.e., Mbp1, Swi4, Swi6, Mcm1, Fkh1, Fkh2, Ndd1, Swi5, and Ace2). The cluster of genes for the gene of interest is called the reference gene cluster (RGC). In an RGC, we assume that each gene shares some cis elements of the gene of interest. Furthermore, the regulatory functions and the interactions of the TFs recognizing the same cis elements are assumed to be the same for all genes in the RGC. For example, in Figure 1a gene MFA2 is the gene of interest; it causes cell cycle arrest and is essential for mating in yeast [19]. This gene has three main cis elements, Ndd1, Mcm1 and Swi5 [4], from which we want to reconstruct the cis-regulatory cir- 


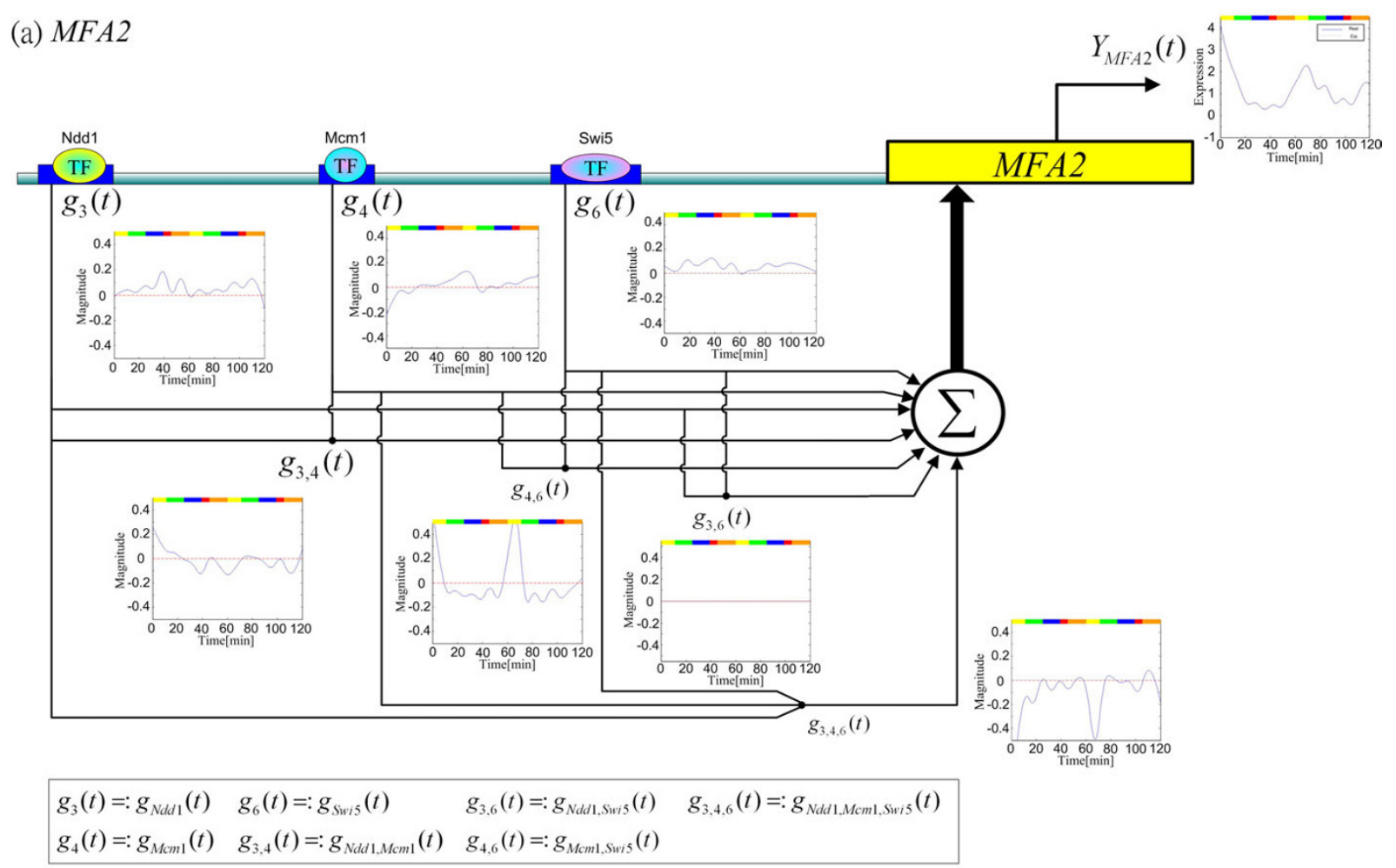

(b) $C L B 2$

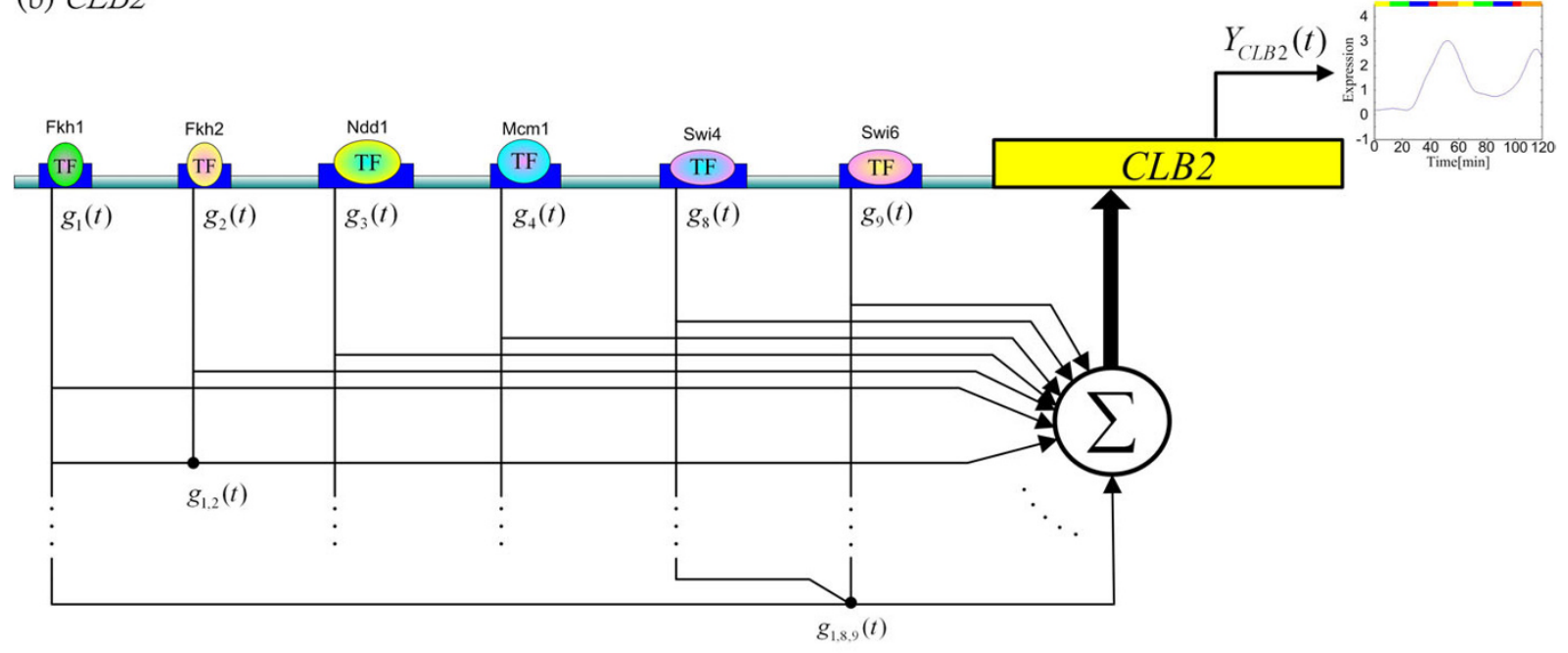

$$
\begin{array}{llll}
g_{1}(t)=: g_{F k h 1}(t) & g_{3}(t)=: g_{N d d 1}(t) & g_{8}(t)=: g_{\text {Swi }}(t) & g_{1,2}(t)=: g_{F k h 1, F k h 2}(t) \\
g_{2}(t)=: g_{F k h 2}(t) & g_{4}(t)=: g_{M c m 1}(t) & g_{9}(t)=: g_{\text {Swi }}(t) & g_{1,8,9}(t)=: g_{F k h 1, \text { Swi } 4 \text {,Swi }}(t)
\end{array}
$$

\section{Figure I}

Dynamic model of the cis-regulatory circuit of gene MFA2 (a) and of gene CLB2 (b). The genome-wide TF-binding location data obtained using chromatin immunoprecipitation [4] is used to identify the transcription factor binding motifs (cis elements). A binding transcription factor $p$ has a regulatory function $g_{p}(t)$ and interacts with other recognizing TFs to produce the regulatory functions $g_{p, q}(t)$ and $g_{p, q, r}(t)$. These regulatory functions are the inputs of the cis-regulatory circuit and generate the dynamic output (i.e., the expression profile) of the target gene. Different phases of the cell cycle are indicated by the colored bar at the right lower corner. 
Table I: The reference gene clusters (RGCs) of MFA2. Target Gene MFA2 and the connectivities to cis elements.

\begin{tabular}{|c|c|c|c|c|c|c|c|c|c|c|c|c|c|c|c|c|c|c|c|}
\hline & FkhI & Fkh2 & NddI & Mcml & Ace2 & Swi5 & Mbpl & Swi4 & Swi6 & & & & & & & & & & \\
\hline MFA2 (YNLI45W) & 0 & 0 & I & I & 0 & I & 0 & 0 & 0 & & & & & & & & & & \\
\hline \multicolumn{20}{|c|}{ Reference genes cluster (RGC) and their connectivities to cis elements } \\
\hline ORF & FkhI & Fkh2 & NddI & Mcm I & Ace2 & Swi5 & Mbpl & Swi4 & Swi6 & ORF & FkhI & Fkh2 & NddI & $\mathrm{Mcm} \mathrm{I}$ & Ace2 & Swi5 & MbpI & Swi4 & Swi6 \\
\hline YAL022C & 0 & 0 & 0 & 0 & 0 & I & 0 & 0 & 0 & YKL209C & 0 & 0 & 0 & 1 & 0 & 0 & 0 & 0 & 0 \\
\hline YAROI8C & 0 & 0 & I & 1 & 0 & 0 & 0 & 0 & 0 & YLR274W & 0 & 0 & 0 & 1 & 0 & 0 & 0 & 0 & 0 \\
\hline YDRI50W & 0 & 0 & I & 0 & 0 & 0 & 0 & 0 & 0 & YML050W & 0 & 0 & 1 & 1 & 0 & 0 & 0 & 0 & 0 \\
\hline YFL026W & 0 & 0 & 0 & 1 & 0 & 0 & 0 & 0 & 0 & YMLI25C & 0 & 0 & 0 & 0 & 0 & 1 & 0 & 0 & 0 \\
\hline YGL032C & 0 & 0 & 0 & 1 & 0 & 0 & 0 & 0 & 0 & YMROOIC & 0 & 0 & 1 & 0 & 0 & 0 & 0 & 0 & 0 \\
\hline YIL050W & 0 & 0 & 0 & 0 & 0 & I & 0 & 0 & 0 & YMR002W & 0 & 0 & I & 0 & 0 & 0 & 0 & 0 & 0 \\
\hline YILI29C & 0 & 0 & 0 & 0 & 0 & 1 & 0 & 0 & 0 & YMR253C & 0 & 0 & 0 & 1 & 0 & 0 & 0 & 0 & 0 \\
\hline YJL079C & 0 & 0 & 1 & 1 & 0 & 0 & 0 & 0 & 0 & YNL056W & 0 & 0 & 1 & 1 & 0 & 0 & 0 & 0 & 0 \\
\hline YJLI57C & 0 & 0 & 0 & 1 & 0 & 0 & 0 & 0 & 0 & YNL058C & 0 & 0 & 1 & 1 & 0 & 0 & 0 & 0 & 0 \\
\hline YKLI63W & 0 & 0 & 0 & 1 & 0 & 1 & 0 & 0 & 0 & YNLI 45W & 0 & 0 & 1 & 1 & 0 & 1 & 0 & 0 & 0 \\
\hline YKLI64C & 0 & 0 & 0 & I & 0 & I & 0 & 0 & 0 & YOR066W & 0 & 0 & 0 & I & 0 & 0 & 0 & 0 & 0 \\
\hline
\end{tabular}

Table 2: The reference gene clusters (RGCs) of CLB2. Target Gene CLB2 and the connectivities to cis elements.

\begin{tabular}{|c|c|c|c|c|c|c|c|c|c|c|c|c|c|c|c|c|c|c|c|}
\hline & FkhI & Fkh2 & NddI & Mcm I & Ace2 & Swi5 & Mbpl & Swi4 & Swi6 & & & & & & & & & & \\
\hline CLB2 (YPR II9W) & 1 & I & 1 & I & 0 & 0 & 0 & I & I & & & & & & & & & & \\
\hline \multicolumn{20}{|c|}{ Reference genes cluster (RGC) and their connectivities to cis elements } \\
\hline ORF & Fkhl & Fkh2 & Nddl & Mcm I & Ace2 & Swi5 & Mbpl & Swi4 & Swi6 & ORF & FkhI & Fkh2 & NddI & $\mathrm{Mcm} \mathrm{I}$ & Ace2 & Swi5 & Mbpl & Swi4 & Swi6 \\
\hline YAROI8C & 0 & 0 & 1 & 1 & 0 & 0 & 0 & 0 & 0 & YKROI3W & 0 & 0 & 0 & 0 & 0 & 0 & 0 & 1 & 1 \\
\hline YBRI33C & 0 & I & 0 & 0 & 0 & 0 & 0 & 0 & 0 & YLR056W & 0 & 0 & 0 & 0 & 0 & 0 & 0 & I & I \\
\hline YBRI38C & 1 & 0 & 1 & 0 & 0 & 0 & 0 & 0 & 0 & YLR084C & 0 & 1 & 1 & 1 & 0 & 0 & 0 & I & 0 \\
\hline YBRI39W & I & 0 & I & 0 & 0 & 0 & 0 & 0 & 0 & YLRI3IC & 0 & 1 & 1 & I & 0 & 0 & 0 & 0 & 0 \\
\hline YCL063W & I & 1 & 0 & 0 & 0 & 0 & 0 & 0 & 0 & YLRI90W & 0 & 1 & 1 & I & 0 & 0 & 0 & 0 & 0 \\
\hline YDL227C & 0 & 0 & 0 & 0 & 0 & 0 & 0 & I & I & YLR209C & I & 0 & 0 & 0 & 0 & 0 & 0 & 0 & 0 \\
\hline YDR033W & 0 & 1 & I & 0 & 0 & 0 & 0 & 0 & 0 & YLR2IOW & I & 0 & 0 & 0 & 0 & 0 & 0 & 0 & 0 \\
\hline YDRI46C & 0 & I & I & I & 0 & 0 & 0 & 0 & 0 & YLR274W & 0 & 0 & 0 & I & 0 & 0 & 0 & 0 & 0 \\
\hline YDRI50W & 0 & 0 & I & 0 & 0 & 0 & 0 & 0 & 0 & YLR342W & 0 & 0 & 0 & 0 & 0 & 0 & 0 & I & 1 \\
\hline YDR224C & 0 & 0 & 0 & 0 & 0 & 0 & 0 & I & I & YML050W & 0 & 0 & 1 & 1 & 0 & 0 & 0 & 0 & 0 \\
\hline
\end{tabular}


Table 2: The reference gene clusters (RGCs) of CLB2. Target Gene CLB2 and the connectivities to cis elements. (Continued)

\begin{tabular}{|c|c|c|c|c|c|c|c|c|c|c|c|c|c|c|c|c|c|c|c|}
\hline YDR225W & 0 & 0 & 0 & 0 & 0 & 0 & 0 & $T$ & 1 & YML064C & 1 & 1 & 0 & 0 & 0 & 0 & 0 & 0 & 0 \\
\hline YDR507C & 0 & 0 & 0 & I & 0 & 0 & 0 & I & I & YMROOIC & 0 & 0 & I & 0 & 0 & 0 & 0 & 0 & 0 \\
\hline YELOI7W & 1 & 0 & 0 & 0 & 0 & 0 & 0 & 0 & 0 & YMR002W & 0 & 0 & 1 & 0 & 0 & 0 & 0 & 0 & 0 \\
\hline YEL040W & 0 & 1 & 0 & 1 & 0 & 0 & 0 & I & I & YMROI5C & 1 & 0 & 0 & 0 & 0 & 0 & 0 & 1 & 0 \\
\hline YEROOIW & 0 & 0 & 0 & 0 & 0 & 0 & 0 & I & I & YMRI83C & 1 & 0 & 0 & 0 & 0 & 0 & 0 & 0 & 0 \\
\hline YFL026W & 0 & 0 & 0 & I & 0 & 0 & 0 & 0 & 0 & YMR253C & 0 & 0 & 0 & 1 & 0 & 0 & 0 & 0 & 0 \\
\hline YGL032C & 0 & 0 & 0 & I & 0 & 0 & 0 & 0 & 0 & YMR305C & 0 & 0 & 0 & 0 & 0 & 0 & 0 & I & I \\
\hline YGL038C & 0 & 0 & 0 & 0 & 0 & 0 & 0 & I & I & YMR307W & 0 & 0 & 0 & 0 & 0 & 0 & 0 & 1 & I \\
\hline YGLII6W & 0 & I & I & 1 & 0 & 0 & 0 & 0 & 0 & YNL056W & 0 & 0 & I & 1 & 0 & 0 & 0 & 0 & 0 \\
\hline YGROI4W & 0 & 0 & 0 & 0 & 0 & 0 & 0 & I & I & YNL058C & 0 & 0 & I & I & 0 & 0 & 0 & 0 & 0 \\
\hline YGR099W & 1 & 0 & 0 & 0 & 0 & 0 & 0 & 0 & 0 & YNL23IC & 0 & 1 & 0 & 0 & 0 & 0 & 0 & 1 & I \\
\hline YGRI5IC & 0 & 0 & 0 & 0 & 0 & 0 & 0 & 1 & 0 & YNL300W & 0 & 0 & 0 & 0 & 0 & 0 & 0 & 1 & I \\
\hline YGRI52C & 0 & 0 & 0 & 0 & 0 & 0 & 0 & I & 0 & YOLOIIW & 0 & 0 & 0 & 0 & 0 & 0 & 0 & 1 & 0 \\
\hline YGRI53W & 0 & 0 & 0 & 0 & 0 & 0 & 0 & 1 & 0 & YOL030W & 1 & 0 & 0 & 0 & 0 & 0 & 0 & 0 & 0 \\
\hline YGR22IC & 0 & 0 & 0 & 0 & 0 & 0 & 0 & I & I & YOLII4C & 0 & 0 & 0 & 0 & 0 & 0 & 0 & I & I \\
\hline YGR279C & 0 & 0 & 0 & 0 & 0 & 0 & 0 & I & I & YOR066W & 0 & 0 & 0 & 1 & 0 & 0 & 0 & 0 & 0 \\
\hline YHR06IC & 0 & I & 0 & 0 & 0 & 0 & 0 & 1 & 1 & YOR073W & 0 & I & 0 & 0 & 0 & 0 & 0 & 0 & 0 \\
\hline YIL056W & 0 & I & I & 0 & 0 & 0 & 0 & I & I & YOR372C & 0 & 0 & 0 & 0 & 0 & 0 & 0 & I & I \\
\hline YILI2IW & 0 & 0 & 0 & 0 & 0 & 0 & 0 & 1 & 0 & YPL032C & 1 & 0 & 0 & 0 & 0 & 0 & 0 & 0 & 0 \\
\hline YILI23W & 0 & I & 0 & I & 0 & 0 & 0 & 1 & 1 & YPLII6W & 1 & 0 & 0 & 0 & 0 & 0 & 0 & 0 & 0 \\
\hline YILI58W & 0 & I & I & I & 0 & 0 & 0 & 0 & 0 & YPLI27C & 0 & 0 & 0 & 0 & 0 & 0 & 0 & I & I \\
\hline YJL05IW & 0 & I & I & 1 & 0 & 0 & 0 & 0 & 0 & YPLI4IC & 1 & 0 & 0 & 0 & 0 & 0 & 0 & 0 & 0 \\
\hline YJL079C & 0 & 0 & I & 1 & 0 & 0 & 0 & 0 & 0 & YPLI55C & 0 & 1 & 0 & 0 & 0 & 0 & 0 & 0 & 0 \\
\hline YJLI57C & 0 & 0 & 0 & 1 & 0 & 0 & 0 & 0 & 0 & YPLI63C & 0 & 0 & 0 & 0 & 0 & 0 & 0 & 1 & I \\
\hline YJLI58C & 0 & I & I & 0 & 0 & 0 & 0 & I & I & YPL255W & 0 & 0 & 0 & 0 & 0 & 0 & 0 & 0 & I \\
\hline YJR054W & 0 & 0 & 0 & 0 & 0 & 0 & 0 & I & I & YPL256C & 0 & 0 & 0 & 0 & 0 & 0 & 0 & 0 & I \\
\hline YJR092W & 1 & 1 & 1 & 1 & 0 & 0 & 0 & 0 & 0 & YPROI3C & 1 & 0 & 0 & 0 & 0 & 0 & 0 & I & 0 \\
\hline YJRIIOW & 0 & I & 0 & 0 & 0 & 0 & 0 & 0 & 0 & YPRII9W & 1 & I & I & 1 & 0 & 0 & 0 & 1 & I \\
\hline YKL096W & 0 & 1 & 0 & 0 & 0 & 0 & 0 & 1 & 1 & YPRI49W & 0 & 1 & 1 & 0 & 0 & 0 & 0 & I & 0 \\
\hline YKLI03C & 0 & 0 & 0 & 0 & 0 & 0 & 0 & I & 0 & YPRI59W & 0 & 0 & 0 & 0 & 0 & 0 & 0 & 1 & I \\
\hline YKL209C & 0 & 0 & 0 & I & 0 & 0 & 0 & 0 & 0 & & & & & & & & & & \\
\hline
\end{tabular}


cuit of MFA2 from yeast microarray data. The cis elements of MFA2 are denoted as follows:

MFA2: $\{\mathrm{Ndd} 1, \mathrm{Mcm} 1$, Swi5 $\}$.

Some genes chosen for the cluster and their cis elements are:

YAL022C: $\{$ Swi5 $\}, \quad$ YAR018C: $\{\mathrm{Ndd} 1, \quad \mathrm{Mcm} 1\}$, YKL163W: $\{\mathrm{Mcm} 1$, Swi5 $\}$....

The cluster of genes can be represented by a connectivity matrix of cis elements as shown in Table 1 in which " 1 " denotes the connection of a cis element with a gene, while "0" means no connection. Similarly, the RGC for gene CLB2 can be represented by the connectivity matrix in Table 2.

\section{Dynamic modeling of cis-regulatory circuits}

Figure 1 illustrates the leaky integrator-based dynamic models of the cis-regulatory circuits of two yeast genes (MFA2 and CLB2) [1,2]. The dynamics of gene expression can be modeled by a simple first-order nonlinear differential equation that is well established and analyzed in [17]; each model includes the possible regulatory functions of the individual TFs and possible interactions among the TFs. For the target gene MFA2 (Figure 1a), the cis-regulatory circuit is modeled by the following dynamic equation

$\dot{Y}_{\text {MFA2 }}(t)=g_{\mathrm{Ndd} 1}(t)+g_{\mathrm{Mcm} 1}(t)+g_{\mathrm{Swi}}(t)+g_{\mathrm{Ndd} 1, \mathrm{Mcm} 1}(t)+$ $g_{\mathrm{Ndd} 1, \mathrm{Swi5}}(t)+g_{\mathrm{Mcm} 1, \mathrm{Swi}}(t)+g_{\mathrm{Ndd} 1, \mathrm{Mcm} 1, \mathrm{Swi}}(t)-\lambda_{\mathrm{MFA2}} Y_{\mathrm{MFA2}}(t)+\varepsilon_{\mathrm{MFA2}}(t)$,

where $\varepsilon_{\mathrm{MFA2}}(t)$ denotes the noise (data uncertainty), $g_{\mathrm{Ndd} 1}(t), g_{\mathrm{Mcm} 1}(t)$ and $g_{\text {Swi5 }}(t)$ are the regulatory functions of transcription factors Ndd1, Mcm1, and Swi5 or the incident transcriptional regulations at the $\mathrm{Ndd} 1$, $\mathrm{Mcm} 1$, and Swi5 cis elements, respectively, $\lambda_{\mathrm{MFA2}}$ represents the mRNA decay rate of the target gene and we used the degradation rate measured by Wang et al. 2002 [20]. The $g_{\mathrm{Ndd} 1, \mathrm{Mcm} 1}(t), g_{\mathrm{Ndd} 1, \mathrm{Swi}}(t), g_{\mathrm{Mcm} 1, \mathrm{Swi}}(t)$ and $g_{\mathrm{Ndd} 1, \mathrm{Mcm} 1, \mathrm{Swi5}}(t)$ denote the following nonlinear interactions among the three TFs:

$g_{\mathrm{Ndd} 1, \mathrm{Mcm} 1}(t)=: f\left(g_{\mathrm{Ndd} 1}(t), g_{\mathrm{Mcm} 1}(t)\right)$,

$g_{\mathrm{Ndd} 1, \text { Swi5 }}(t)=: f\left(g_{\mathrm{Ndd} 1}(t), g_{\mathrm{Swi5}}(t)\right)$,

$g_{\mathrm{Mcm} 1, \text { Swi5 }}(t)=: f\left(g_{\mathrm{Mcm} 1}(t), g_{\text {Swi5 }}(t)\right)$,

and

$g_{\mathrm{Ndd} 1, \mathrm{Mcm} 1, \mathrm{Swi}}(t)=: f\left(g_{\mathrm{Ndd} 1}(t), g_{\mathrm{Mcm} 1}(t), g_{\mathrm{Swi5}}(t)\right)$.

The biological meaning of Equation (2) is that the change in the mRNA expression level of gene MFA2 is due to the productions of regulatory functions of individual TFs and interactions among the TFs, i.e., $g_{\mathrm{Ndd} 1}(t)+g_{\mathrm{Mcm} 1}(t)+$ $g_{\mathrm{Swi}}(t)+g_{\mathrm{Ndd} 1, \mathrm{Mcm} 1}(t)+g_{\mathrm{Ndd} 1, \mathrm{Swi}}(t)+g_{\mathrm{Mcm} 1, \mathrm{Swi}}(t)+$ $g_{\mathrm{Ndd1}, \mathrm{Mcm} 1, \mathrm{Swi5}}(t)$, and $-\lambda_{\mathrm{MFA2}} Y_{\mathrm{MFA} 2}(t)$, which is the degradation of mRNA. Similarly, the cis-regulatory circuit of the target gene CLB2 in Figure 1b is modeled by

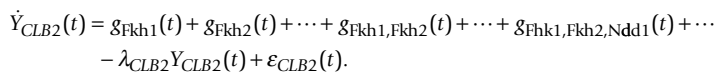

For simplicity, the indices of target genes and cis elements are denoted by numerical notation, so that the cis-regulatory circuit of gene $i$ can be written as

$$
\dot{Y}_{i}(t)=\sum_{p}^{v} g_{p}(t)+\sum_{p q} g_{p, q}(t)+\sum_{p q r} g_{p, q, r}(t)+\cdots-\lambda_{i} Y_{i}(t)+\varepsilon_{i}(t),
$$

where $v$ is the total number of cis elements in gene $i$ and its corresponding degradation rate $\lambda_{i}$ measured by Wang $e t$ al. 2002 [20]. If $\lambda_{i}$ is unavailable, it should be estimated together with the parameters $g_{p}(t), g_{p, q}(t), g_{p, q, r}(t)$ (see Methods). For the cis-regulatory circuits in Equations (2), (5) and (6), one obviously cannot estimate the multiple unknowns $g_{p}(t), g_{p, q}(t), g_{p, q, r}(t), \ldots$ by only the expression profile (i.e., $\left.Y_{i}(t)\right)$ of the $i^{\text {th }}$ target gene. However, since the functions $g_{p}(t), g_{p, q}(t), g_{p, q, r}(t), \ldots$ are assumed to be the same for all genes in the RGC and since there are overlaps of cis elements among genes in this RGC, one can estimate these functions from an array of expression profiles $Y_{1}(t)$, $Y_{2}(t), \ldots, Y_{N}(t)$ of the genes in the RGC simultaneously, taking advantage of cross information enhancement. The RGCs for MFA2 and CLB2 are shown in Table 1 and Table 2 , respectively. In this situation, a cross-gene identification method is proposed as follows. By integrating the dynamic equations of cis-regulatory circuits for $N$ genes in the RGC of the gene of interest, we obtain the following array of dynamic equations

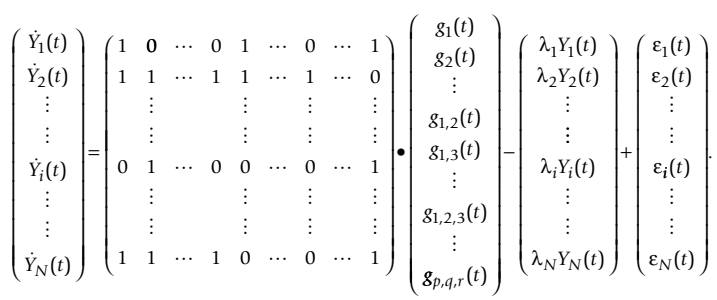

In the cross-gene dynamic equations in (7), the process to identify regulatory functions $g_{p}(t), g_{p, q}(t)$ and $g_{p, q, r}(t)$ from microarray data $Y_{i}(t), i=1,2, \ldots, N$ is called the cross-gene identification approach, in which the regulatory functions $g_{p}(t), g_{p, q}(t)$ and $g_{p, q, r}(t)$ are shared by genes in the RGC. Therefore, the estimation of the regulatory functions of 


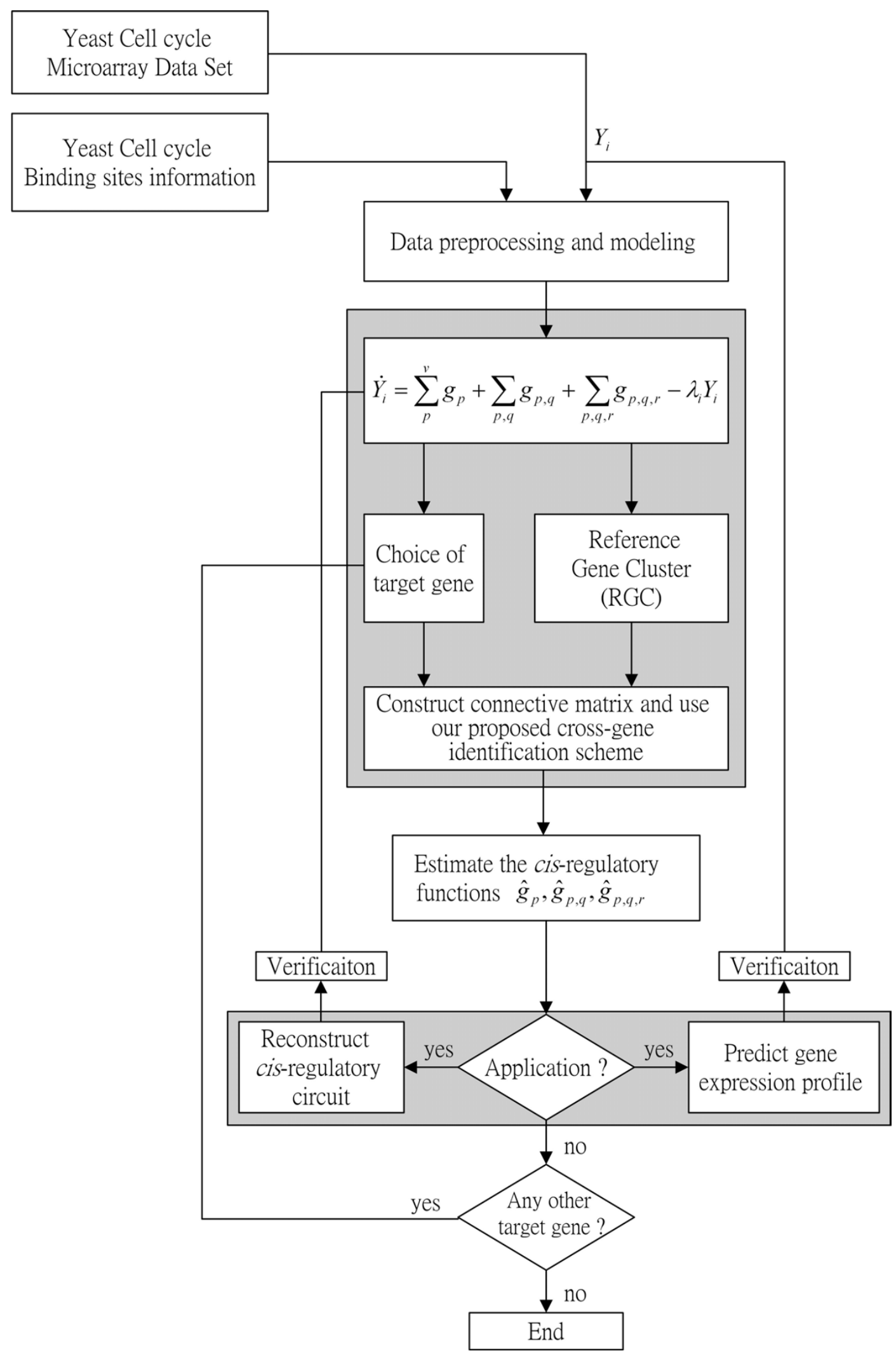

Figure 2

The overall flowchart of the modeling, identification and prediction of a cis-regulatory circuit. 
All possible cis-regulatory functions of gene CLB2
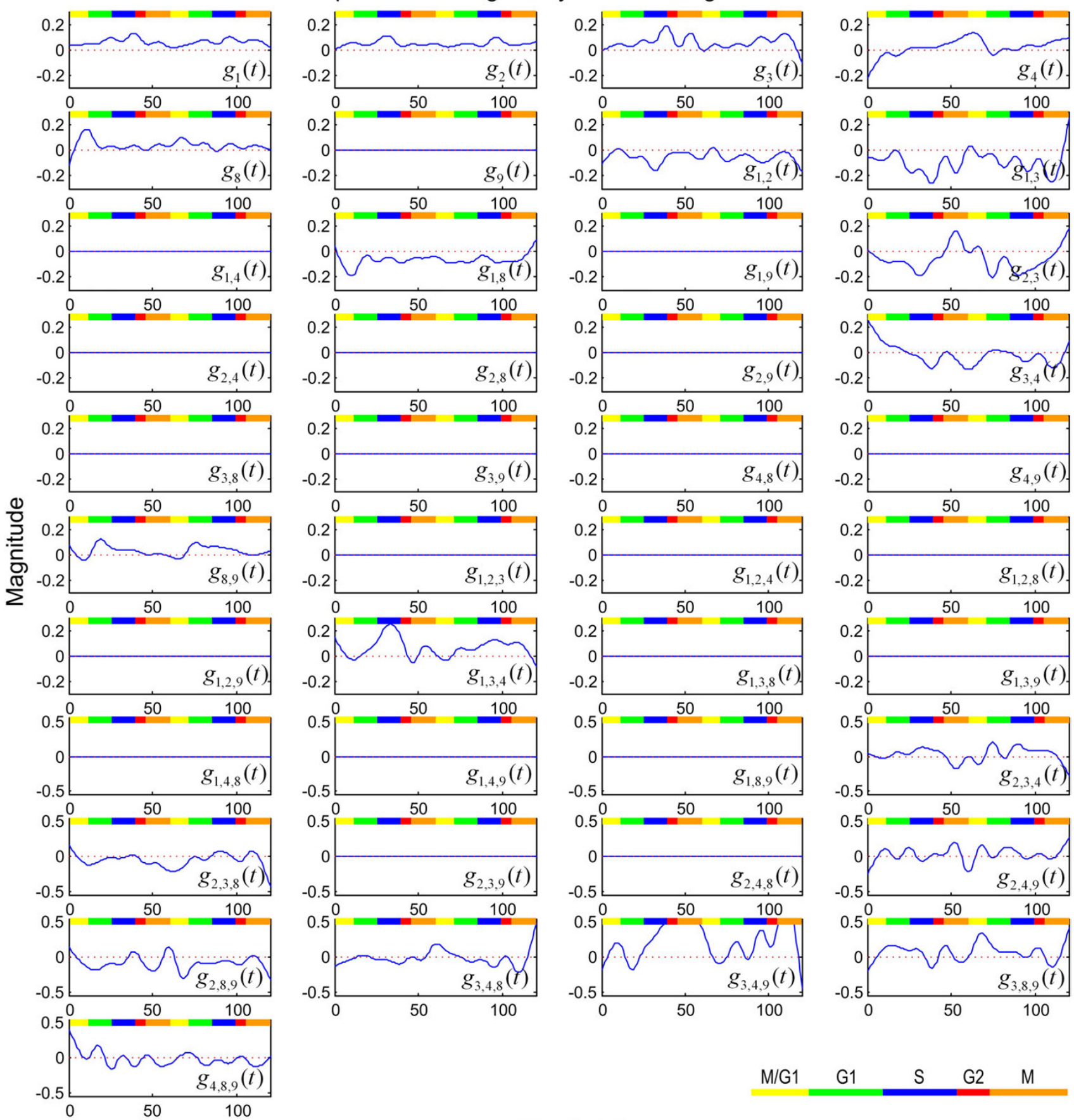

Time[min]

$$
\begin{array}{llll}
g_{1}(t)=: g_{\mathrm{Fkh} 1}(t) & g_{3}(t)=: g_{\mathrm{Ndd} 1}(t) & g_{8}(t)=: g_{\mathrm{Swi} 4}(t) & g_{1,2}(t)=: g_{\mathrm{Fkh} 1, \mathrm{Fkh} 2}(t) \\
g_{2}(t)=: g_{\mathrm{Fkh} 2}(t) & g_{4}(t)=: g_{\mathrm{Mcm} 1}(t) & g_{9}(t)=: g_{\mathrm{Swi} 6}(t) & g_{1,3,4}(t)=: g_{\mathrm{Fkh} 1, \mathrm{Ndd} 1, \mathrm{Mcm} 1}(t)
\end{array}
$$

Figure 3

All estimated cis-regulatory functions, including the regulatory function $g_{p}(t)$ of each individual TF and the interactions $g_{p, q}(t)$ and $g_{p, q, r}(t)$ among the TFs that recognize the cis elements of the CLB2 gene. The numerical notation of regulatory functions is given in the box at the bottom of the figure. Different phases in the cell cycle are indicated by the colored bar near the right lower corner. 
one gene can also use the information from the profiles $Y_{1}(t), Y_{2}(t), \ldots, Y_{N}(t)$ of other genes in RGC to improve the identification ability of the regulatory functions to reconstruct the cis-regulatory circuit of the gene of interest, which is called cross information enhancement.

Remark 1 : Suppose that the gene of interest in Equation (6) has cis elements $p=1, \ldots, v$. Then all genes whose cis elements are subsets of these $v$ cis elements are included in the same RGC of the gene of interest.

\section{Cross-gene identification scheme}

Since the number of functions $g_{p}(t), g_{p, q}(t), g_{p, q, r}(t), \ldots$ is finite, we can estimate these functions if the number $\mathrm{N}$ of dynamic equations in Equation (7) is large enough. Equation (7) can be rewritten in an algebraic form

$\mathrm{X}(t)=\mathrm{A} \cdot \mathrm{G}(t)+\mathrm{E}(t)$

where

$$
\mathrm{X}(t)=\left(\begin{array}{c}
\dot{Y}_{1}(t)+\lambda_{1} Y_{1}(t) \\
\dot{Y}_{2}(t)+\lambda_{2} Y_{2}(t) \\
\vdots \\
\vdots \\
\dot{Y}_{i}(t)+\lambda_{i} Y_{i}(t) \\
\vdots \\
\vdots \\
\dot{Y}_{N}(t)+\lambda_{N} Y_{N}(t)
\end{array}\right), \quad \mathrm{G}(t)=\left(\begin{array}{c}
g_{1}(t) \\
g_{2}(t) \\
\vdots \\
g_{1,2}(t) \\
g_{1,3}(t) \\
\vdots \\
g_{1,2,3}(t) \\
\vdots \\
g_{p, q, r}(t)
\end{array}\right), \quad \mathrm{E}(t)=\left(\begin{array}{c}
\varepsilon_{1}(t) \\
\varepsilon_{2}(t) \\
\vdots \\
\vdots \\
\varepsilon_{i}(t) \\
\vdots \\
\vdots \\
\varepsilon_{N}(t)
\end{array}\right)
$$

Remark 2 : In order to calculate the derivatives in $\mathrm{X}(t)$ from undersampled data, a cubic spline interpolation method $[21,22]$ is employed for curve fitting to obtain more accurate differential values and to learn more reliable models.

In Equation (8), $\mathrm{X}(t)$ can be calculated from microarray data for the RGC of the gene of interest, which can then be used to estimate the vector $\mathrm{G}(t)$ by the least squares method, leading to the following solution:

$$
\hat{\mathrm{G}}(t)=\left(\mathrm{A}^{T} \mathrm{~A}\right)^{-1} \mathrm{~A}^{T} \mathrm{X}(t)
$$

for all $t$. After $\hat{\mathrm{G}}(t)$ is estimated from Equation (9), the regulatory functions $g_{1}(t), \ldots, g_{1,2}(t), \ldots$ and $g_{p, q, r}(t)$ in Equation (8) can be reconstructed for the genes in the RGC at every time point. If a cis-regulatory circuit is free of any function $g_{p}(t), g_{p, q}(t)$, or $g_{p, q, r}(t)$, the value of the estimated function should be very small or zero. After the functions are estimated, they can be plugged into Equa- tion (6) and the reconstruction of the cis-regulatory circuit of the gene of interest is completed. The flowchart for modeling, identifying and predicting a cis-regulatory circuit is shown in Figure 2.

In order to obtain more accurate cis-regulatory circuits, the model should include the triple interactions among the recognizing TFs; i.e., the vector $\mathrm{G}(t)$ in Equation (7) should include $g_{p, q, r}(t)$.

Remark 3: If the degradation parameters $\lambda_{i}$ in (6) are unavailable, the estimation procedure of $\mathrm{G}(t)$ and $\lambda_{i}$ from equation (7) to equation (9) should be modified as in Methods.

\section{Two application examples}

I. The cis-regulatory circuit of MFA2

Suppose that the cis-regulatory circuit of the MFA2 gene is of interest. We construct a dynamic model of the cis-regulatory circuits of the genes in the RGC of MFA2 in Table 1 and then estimate the regulatory functions and interactions by the cross-gene identification scheme. The estimated transcriptional regulatory functions $g_{p}(t)$ and interactions $g_{p, q}(t)$ and $g_{p, q, r}(t)$ are shown as the insets in Figure 1a. These insets indicate that cis elements Ndd1, $\mathrm{Mcm} 1$ and Swi5 in MFA2 all have cell cycle regulatory abilities in the late G1 phase. In addition, every individual cis element has a positive regulatory function on the MFA2 gene; for example, the function $\mathrm{g}_{4}(\mathrm{t})$ for $\mathrm{Mcm} 1$ has an obvious peak value in the transition phase late G1 of the cell cycle. Michaelis and Herskowitz [19] found that the MFA2 gene causes the cell cycle arrest at the G1 phase and is required for mating in yeast. Note that the interaction $g_{3,6}(t)$ between TFs Ndd1 and Swi5 is very weak or absent in the cell cycle. In contrast, the interaction $\mathrm{g}_{4,6}(\mathrm{t})$ between TFs Mcm 1 and Swi5 is dynamic; it has a high positive peak value in the late $\mathrm{G} 1$ phase, which coincides with MFA2's activity phase. This interaction seems to play an important role of positive regulation in this cis-regulatory circuit. On the other hand, the regulatory function $g_{3,4,6}(t)$ of the interaction among TFs Ndd1, Mcm 1 and Swi5 is negative on gene MFA2. This regulation may repress the expression of gene MFA2 to make the expression decay to the steady state. If there is no repression function such as $\mathrm{g}_{3,4,6}(\mathrm{t})$, the expression of MFA2 will increase and may disrupt in the cell cycle.

\section{The cis-regulatory circuit of CLB2}

$\mathrm{Clb}$ proteins are crucial cyclins for completing the G2/M transition of the mitotic cell cycle and the most typical one is the B-type mitotic cyclin Clb2, which is required for entry into mitosis [23]. Suppose that the cis-regulatory circuit of gene CLB2 is of interest. Fkh1, Fkh2, Ndd1, Mcm1, Swi4 and Swi6 have been identified as the TFs that bind to the promoter sequence of CLB2 $[3,4,24,25]$. As shown in 

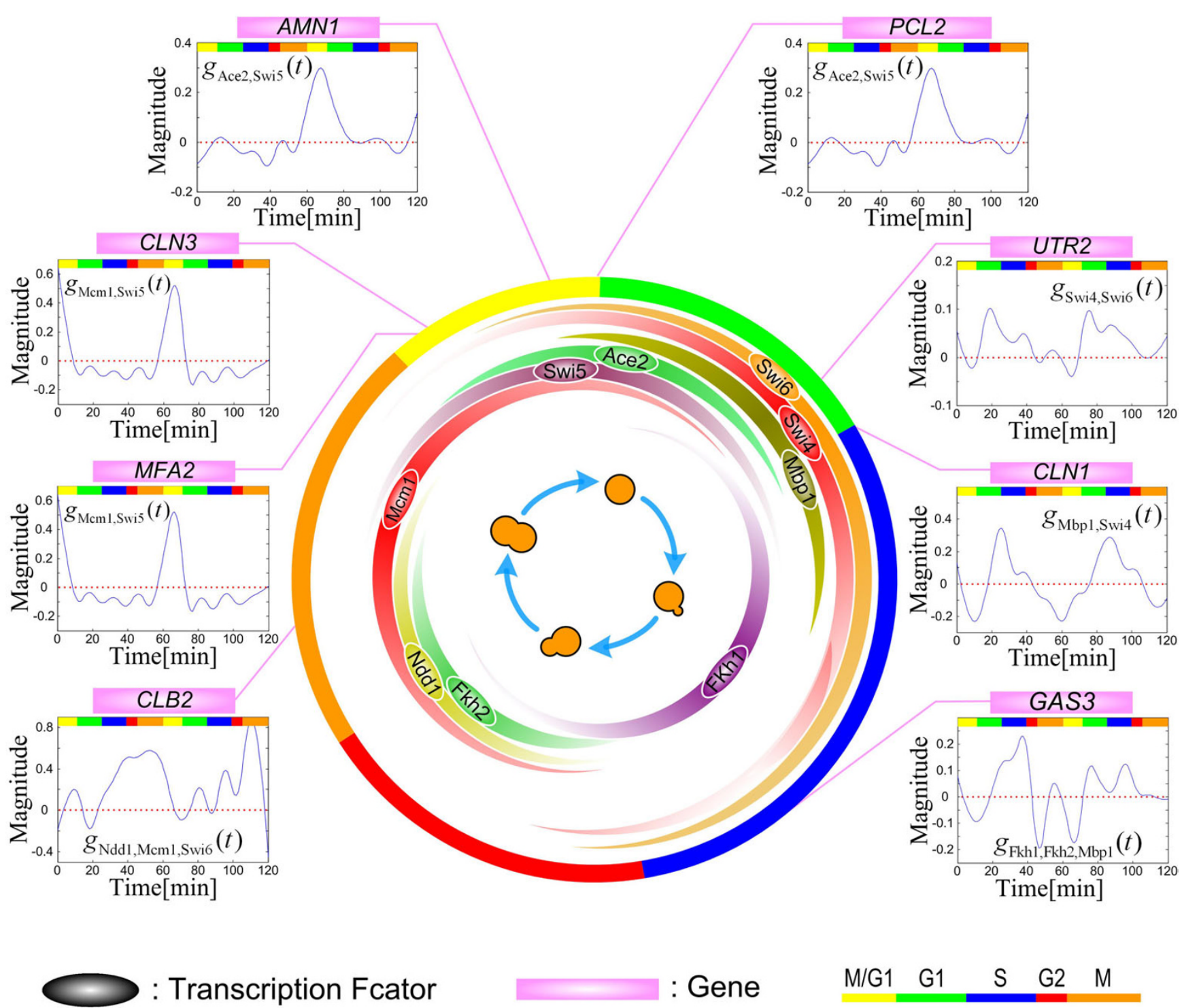

: Gene

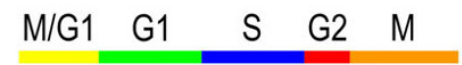

\section{Figure 4}

The gene expression phases match the main regulatory functions of TFs. It is seen that the main interaction functions of TFs have a peak value and always occur during or soon after the mRNA expression phases of the corresponding genes. For example, the gene MFA2 has the main interaction regulatory function $g_{M c m l, S w i 5}(t)$ which has a peak during the expression phases between the TFs Mcm I and Swi5 by identifying the cis-regulatory circuit. As another example, the gene UTR2 has the main interaction regulatory function $g_{S w i 4, S w i b}(t)$, which has a peak during the expression phases between the TFs Swi4 and Swi6 by identifying the cis-regulatory circuit. These results indicate that the main regulatory functions have a peak value phase to match the gene expression phase. Therefore, we can estimate the gene expression phase by identifying the main regulatory function. The width of a colored band in the inner circle is approximately proportional to the expression level of the TF gene of interest in the cell cycle. A pink line points to the main expression phase of a target gene in the pink box. Different phases in the cell cycle are indicated by the colored bar at the right lower corner.

Figure $1 \mathrm{~b}$, the possible cis-regulatory circuit of CLB2 is very complex. Using the cross-gene identification scheme, we reconstructed the cis-regulatory functions shown in Figure 3 . Although there are 41 possible regulatory functions, including $g_{p}(t), g_{p, q}(t)$ and $g_{p, q, r}(t)$, only 20 regula- tory functions are found to have nonzero values (Figure $3)$.

The two cis elements Fkh1 and Fkh2 are found to have very similar regulatory functions $g_{1}(t)$ and $g_{2}(t)$, in agree- 

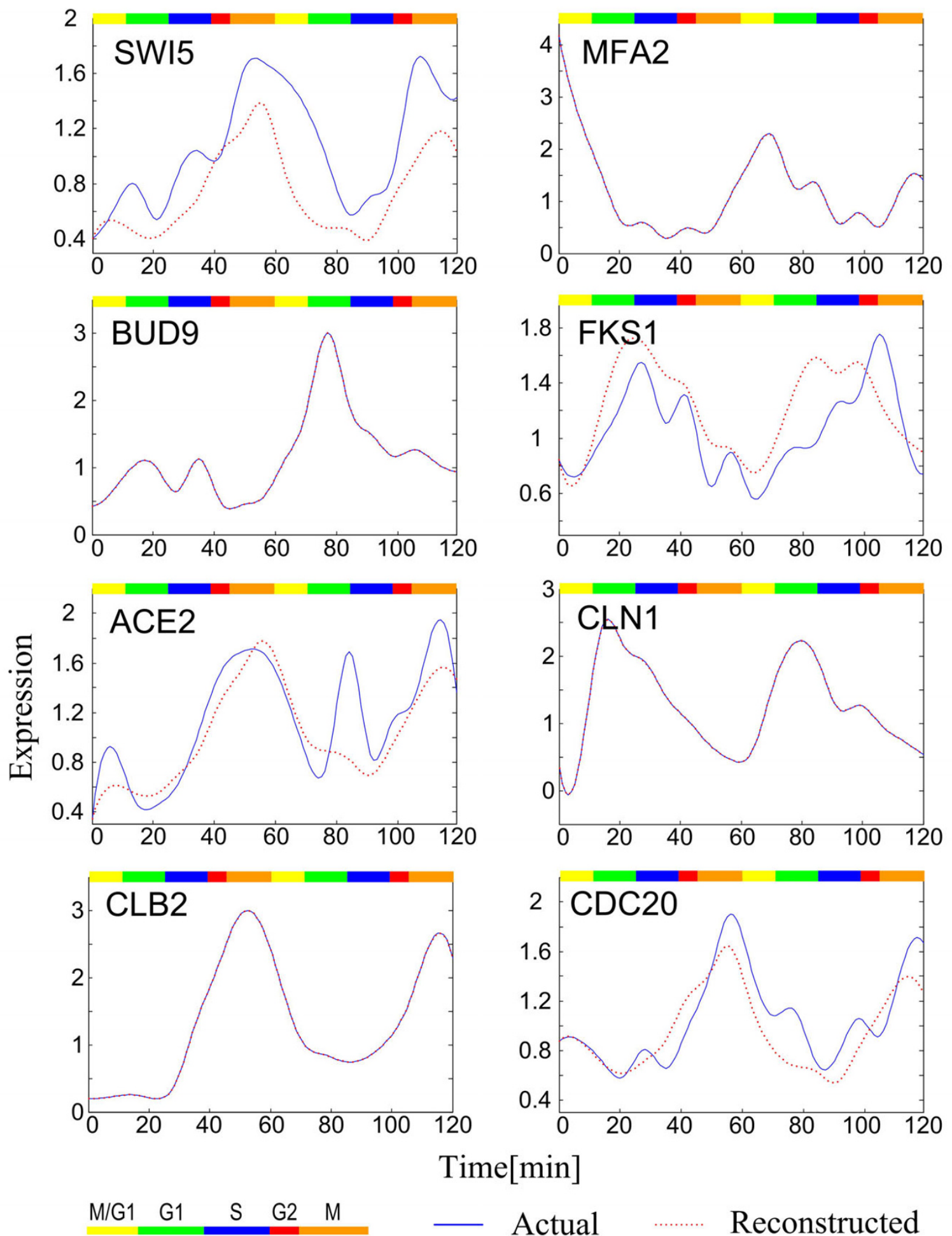

\section{Time[min]}

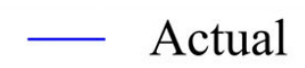

\section{Reconstructed}

\section{Figure 5}

Comparison between the actual gene expression profiles (with cubic spline) and the reconstructed gene expression profiles. The examples shown were randomly chosen. The reconstructed gene expression profiles were obtained by integrating the estimated cis-regulatory functions and the chromatin immunoprecipitation data. When there is only one blue line in a figure it means that the reconstructed function is very close to the actual gene expression profile. Different phases in the cell cycle are indicated by the colored bar. 
ment with the experimental evidence that the forkhead family members Fkh1 and Fkh2 of transcription factors have overlapping roles in the control of the G2/M transition $[25,26]$. The regulatory function $g_{2}(t)$ of Fkh2 has a distinct cell cycle regulatory ability (Figure 3 ) and especially, the interaction function $g_{2,3}(t)$ between Fkh2 and Ndd1 has a strong regulatory contribution to the gene expression profile in the $\mathrm{M} / \mathrm{G} 1$ phase. The regulatory function $g_{4}(t)$ of $\mathrm{Mcm} 1$ has two peaks. There is experimental evidence that $\mathrm{Mcm} 1$ is a member of an evolutionarily conserved class of transcription factors that have related to DNA binding sequences and dimerization domains. In addition, $\mathrm{Mcm} 1$ binds the early cell cycle box (ECB) that contains a Mcm1 cis element in the SWI4, CLN3, CDC6, and CDC47 promoters and activates M/G1-specific transcription [27].

The cell cycle genes that are activated during the late G1 or $S$ phase have SBF or MBF sequence-specific transcription factors that bind the cis elements in their promoter region. SBF (the Swi4-Swi6 cell cycle box binding factor) is a heterodimer of Swi4 and Swi6 $[3,28,29]$. The regulatory functions $g_{8}(t)$ and $g_{9}(t)$ of Swi4 and Swi6 and their interaction function $g_{8,9}(t)$ are estimated using the dynamic expression model (Figure 3). It is well-known that neither Swi4 nor Swi6 alone has obvious cell cycle regulation ability, and indeed we found that $g_{8}(t)$ has only one peak and so shows no cycle and that $g_{9}(t)$ shows no capability of cell cycle regulation (Figure 3). In contrast, the combination of Swi6 and Swi4 to make the complex SBF enables the cis elements Swi6 and Swi4 to provide cell cycle regulation capacity; that is, the interaction function $g_{8,9}(t)$ of Swi6 and Swi4 has a peak during the G1/S phase of the cell cycle. Ndd1 and Fkh2 are bound to identical promoters throughout the cell cycle and their interaction $g_{2,3}(t)$ is an important transcriptional process targeted by the $\mathrm{Cdk}$ activity $[24,30]$. In addition, there is another obvious positive interaction $g_{3,4,9}(t)$ among Ndd1, Mcm1, and Swi6 (Figure 3). It has a large regulatory ability in the G1/S phase, which almost dominates the expression profile of $C L B 2$. In contrast, $g_{3,4}(t)$ has a negative regulation contribution. We therefore propose that the key factor Swi6 in the interaction $g_{3,4,9}(t)$ is similar to its role in SBF and MBF. At any rate, our model suggests that Swi6 plays a key role in the interaction among $\mathrm{Ndd} 1, \mathrm{Mcm} 1$, and Swi6. This is a new finding in the cis-regulatory circuit of CLB2.

We also confirm the well-known interaction among $\mathrm{Ndd} 1$, Fkh2, and $\mathrm{Mcm} 1$ in the cis-regulatory circuits of the CLB2 and SWI5 genes because the interaction function $g_{2,3,4}(t)$ has a distinct regulatory ability in the G2/M phase of the cell cycle (Figure 3). Interestingly, the interaction function $g_{3,4,9}(t)$ among Ndd1, Mcm1 and Swi6 is about two times higher than any of the other functions in Figure 3. In addition, the interaction $g_{1,3,4}(t)$ among Fkh1, Ndd1 and $\mathrm{Mcm} 1$ is highly positive, the interaction $g_{3,4,8}(t)$ among Ndd1, Mcm 1 and Swi4 is mildly positive, while the interaction $g_{3,8,9}(t)$ among Ndd1, Swi4 and Swi6 is negative. These observations are in agreement with the fact that the regulatory ability of an interaction among TFs is usually much stronger than that of an individual TF; in other words, there is synergy among TFs.

In summary, there are many experimental observations that support the cis-regulatory functions identified by the dynamic model and our model provides novel insights into the quantitative regulation of the cis-regulatory circuit of a gene of interest.

\section{Support from expression phases of TFs in the cell cycle} In this paper, the question of why the strengths of regulatory functions in the cis-regulatory circuits are different in different phases of the cell cycle is investigated. Based on the mRNA expression profiles of transcription factor genes from experiments, the distribution of the expressions of the nine TF genes in the different phases of the cell cycle is shown in Figure 4. In support of our results, the large positive interaction functions (peaks) among a set of TFs always occur during the expression phases of the genes of the interacting TFs. For example, for the cis-regulatory circuit of MFA2 (Figure 1a), there is an obvious peak for the function $\mathrm{g}_{4,6}(\mathrm{t})$ of the interaction between TFs $\mathrm{Mcm} 1$ and Swi5 during the $M$ phase, and in Figure 4, this peak indeed occurs during the expression phases of the two TF genes $[18,27]$. As another example, for the cis-regulatory circuit of CLB2, there is a strong interaction $\left(\mathrm{g}_{3,4,9}\right.$ (t)) among Ndd1, Mcm1 and Swi6 starting from the G2 phase (Figure 3 ). We can therefore infer that the expression of a cell cycle gene in a specific phase of the cell cycle needs a specific inducing signal, which is mainly from the interactions of certain specific TFs that bind the cis elements of the gene.

\section{Accuracy of reconstructed cis-regulatory circuits}

The accuracy of the reconstructed cis-regulatory circuit of a gene can be evaluated by reconstructing the expression profile of the gene using the reconstructed cis-regulatory circuit

$\dot{\hat{Y}}_{i}(t)=\sum_{p}^{v} \hat{g}_{p}(t)+\sum_{p q} \hat{g}_{p, q}(t)+\sum_{p q r} \hat{g}_{p, q, r}(t)+\cdots-\lambda_{i} \hat{Y}_{i}(t)$,

where $\hat{g}_{p}(t), \hat{g}_{p, q}(t)$ and $\hat{g}_{p, q, r}(t)$ have been estimated by the cross-gene iaentification scneme. The reconstructed profile and the observed profile are compared in Figure 5. We find that if the number of the cis elements of a gene is large enough, the reconstructed expression profile is very accurate; otherwise, it may be inaccurate. Fortunately, although the reconstructed expression profile is not accu- 
a

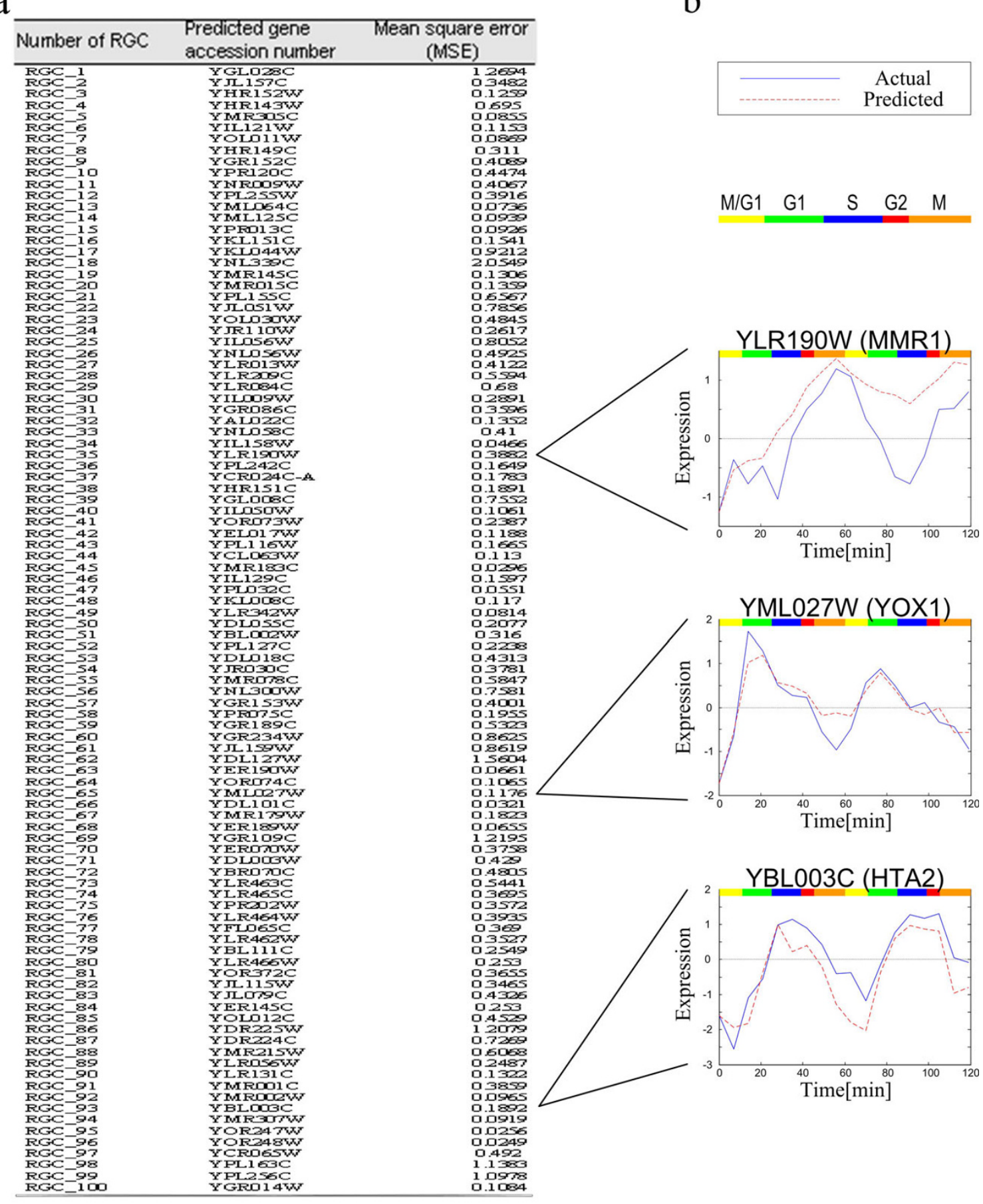

\section{Figure 6}

The regulatory functions integrated with ChIP-chip data to predict gene expression profiles. To test the prediction performance of our model, (a) 100 yeast cell cycle genes that have not been employed in the reconstruction of the cis-regulatory circuits are randomly chosen from their corresponding reference gene clusters (RGCs). The maximum mean square error (MSE) of prediction results is 2.055 and the minimum is 0.025 . (b) Three examples of the comparison between the actual (blue) and the predicted (red) gene expression profiles. Different phases in the cell cycle are indicated by the colored bar. 
rate in some genes, the trend of the expression profile for a gene is always correct.

\section{Prediction of gene expression profile}

In the above, each regulatory circuit was identified using $95 \%$ of genes in its RGC, and the remaining $5 \%$ of genes in the RGC will now be used for predicting expression profiles, i.e., for cross validation. Our cross-gene identification scheme assumes that the regulatory functions of TFs are universal in the cluster of genes with similar functions. Under this assumption, our method should be able to predict the expression profiles of other genes in the same cluster that have not been employed in Equation (7) to reconstruct the cis-regulatory circuits. This is one way to validate our model.

In Figure 6, we randomly chose 100 RGCs to test the prediction accuracy; that is, for each RGC we show the prediction result for one of the unused genes. Figure 6a shows the predicted target gene and the mean square error (MSE) of prediction results which has the maximum of 2.055 and the minimum of 0.025 . In addition, three examples of the detailed comparison between the actual and the predicted gene expression profiles are shown in Figure 6b. In general, the predicted profiles are satisfactory approximations of the observed expression profiles. We found that the smaller the number of the cis elements, the less accurate the prediction results. However, if some cis elements of a gene have strong regulatory functions, the expression profiles of this gene can be predicted accurately even when the number of cis elements is small. If some genes in the RGC have the same cis elements but have different observed gene expression profiles, these expression profiles will lead to poor estimation of parameters. This is the main cause of prediction error. Why does this situation arise? It may be that some cis elements of these genes have not yet been identified or there are some errors in the inference of the cis elements. For example, MMR1 may have another cis element Gcr2 [5] and this may be why the predicted profile is quite different from the observed profile (Figure 6b).

\section{Discussion}

In contrast to current methods, our method uses all possible expression profile information from the cluster of genes to reconstruct the cis-regulatory circuit of a target gene. In particular, our method is capable of extracting dynamic interactions among TFs. For this reason, the analysis and interpretation of output expression profiles become straightforward. Therefore, our method has a high potential for applications such as studying variations of the cis-regulatory circuit of the same gene in different yeast strains to investigate the regulatory evolution of the gene.
The contributions of this study include: (1) a nonlinear dynamical model is developed for cis-regulatory circuits in terms of regulatory functions and interactions among TFs, (2) a cross-gene identification scheme is proposed to estimate many parameters involved in the dynamical model of cis-regulatory circuits from the expression profiles of genes in the reference gene cluster, (3) a detailed identification of the dynamic cis-regulatory abilities of TFs, which vary with time, and (4) a gene expression prediction method is developed by the proposed dynamic cis-regulatory circuit, assuming that the cis-regulatory functions of the same TFs in different circuits are the same. Three advantages of our method over current methods are that the cis-regulatory circuit is constructed with the circuit structure intact, that it uses the expression profiles of many genes simultaneously to obtain extra information, and that it is dynamic and quantitative.

Significantly, our model not only can confirm known regulations but also can provide conjectures for experimental verification. Consider the key positive cis-regulatory function $g_{4,6}(t)$ in Figure 1a. We propose that during the expression of gene MFA2, the transcription factor Ndd1 (the G2/M phase) communicates with the transcription factor $\mathrm{Mcm} 1$ (the $\mathrm{M}$ phase) to transmit a specific signal to induce the expression of the MFA2 gene. Such conjectures from the reconstructed cis-regulatory circuits may be useful for studying the regulatory evolution of genes by comparing the cis-regulatory functions of different strains, or for predicting the gene expression behavior before conducting an experiment.

However, we found poor results in some cases. For example, in Figure 3, we were unable to find the basal regulatory function $g_{9}(t)$ of individual Swi6 or the interaction $g_{2,4}(t)$ between Fkh2 and $\mathrm{Mcm} 1$ for gene CLB2 [31]. These regulatory functions have not been identified by our scheme because they have no obvious specific phase regulatory ability. Besides, from the cis-regulatory functions in Figures 1 and 2, several cis-regulatory function profiles did not show a clear periodicity. A possible reason may be that the original microarray data were noisy and the use of cubic spline interpolation and linear transformation of microarray data in our scheme had introduced new noise and distortions. Most probably, the cis element information used to construct the cis-regulatory circuits under yeast cell cycle is not complete; only nine significant $c$ is elements were considered in this study to reduce the complexity of the mathematical model. Another possible source of error is that we have not considered the order of the cis elements on the promoter region, which may affect the strength of the interaction between TFs [36]. Such differences, however, can be incorporated by putting, say both $g_{p, q}(t)$ and $g_{p, q, r}(t)$, into the model. 
In view of the facts that there are uncertainties about the cis elements of some of the genes studied and that microarray data are noisy, it is remarkable that our method gave accurate results for the expression profiles of the majority of the cell cycle genes studied and also gave fairly accurate predictions of the expression profiles of other cell cycle genes. In the future, if better cis elements data and more accurate and longer gene expression profile data become available, we should be able to improve the reconstruction of cis-regulatory circuits. Also, our approach may be extended to reconstruct cis-regulatory circuits in more diverse conditions and more complex eukaryotes. After cis-regulatory circuits are accurately described by explicit dynamical equations, some applications will be straightforward.

\section{Conclusion}

In this study, we assume that the regulatory functions of the same cis elements and the interaction functions among their TFs are similar across genes within the cluster of genes with overlapping cis elements; i.e., the regulatory functions and interaction functions are universal in this cluster of genes. Under this assumption, the cross-gene identification scheme takes advantage of cross-information enhancement to improve the accuracy of parameter estimation. The number of genes used should be large enough, so that we have a large number of outputs (i.e., their microarray data) for parameter estimation.

After the parameters of the cis-regulatory circuits of interest have been estimated, the circuits can be explicitly described by plugging these parameters into their corresponding dynamic equations. Moreover, these estimated functions and interactions can be used to predict the expression profiles of other genes that share the same cisregulatory elements but have not been used to identify the cis-regulatory circuits. In this manner, we can evaluate the performance of the proposed dynamic model of cis-regulatory circuits. From a number of examples, we have found that the predicted results are in most cases satisfactory, confirming the validity of the proposed dynamic model of cis-regulatory circuits. Our modeling represents a new approach to studying cis-regulatory circuits from cross-gene expression data. It is a systems biology approach because we consider the regulatory circuits of many genes and many TFs at the same time and we use system identification techniques to estimate the parameters of the circuits. The results of expression prediction from experimental data suggest that our novel approach is suitable for deciphering the regulatory functions and the cooperativity of the TFs that regulate the expression of a gene.

\section{Methods \\ Experimental data}

To identify the cis-regulatory circuit of a gene of interest in the yeast cell cycle, we apply our approach to the data of Spellman et al. 1998 [18], which contains expression profiles of 6178 open reading frames (ORFs) in the yeast Saccharomyces cerevisiae during the cell cycle [33]. Our analysis was applied to the $\alpha$-factor arrest data set. The raw data were transformed into a linear scale from the original log ratio carried out by Spellman et al. 1998 [18]. To reduce the effect of noise and to overcome undersampled microarray data in the estimation of cis-regulatory circuits, the cubic spline was used for data interpolation and smoothing to obtain a less sensitive first derivative of the expression pattern and to learn a more reliable model. Furthermore, the noise is modeled in the noise term $\varepsilon_{\mathrm{i}}(\mathrm{t})$ in Equation (7).

From the RGC of the gene of interest, the cross-gene identification method from Equations (8) to (9) is employed to reconstruct the cis-regulatory circuit. The connectivity information between TFs and their target genes was obtained from the yeast cell cycle analysis [4]. We focused on the nine transcription factors that have been identified to play important roles in the transcription regulation of a set of yeast genes whose expressions are cell-cycle dependent; these nine transcription factors are Mbp1, Swi4, Swi6, Mcm1, Fkh1, Fkh2, Ndd1, Swi5, and Ace2 $[24,26,27,34]$ (See additional file 1: Table for the original data used to perform this analysis).

\section{Estimation of degradation rate}

If the mRNA degradation rate $\lambda_{\mathrm{i}}$ in Equation (6) has not been estimated experimentally, it should be estimated together with the parameters $g_{p}(t), g_{p, q}(t), g_{p, q, r}(t)$,. The algorithm to estimate the $\lambda_{\mathrm{i}}$ is described as follows. First, Equation (6) is changed to

$$
\dot{Y}_{i}(t)=\sum_{p}^{v} g_{p}(t)+\sum_{p q} g_{p, q}(t)+\sum_{p q r} g_{p, q, r}(t)+\cdots-\lambda_{i}(t) Y_{i}(t)+\varepsilon_{i}(t)
$$

where $v$ is the total number of cis elements in gene $i$, and the degradation rate is substituted as $\lambda_{i}(t)$. Similarly, since the functions $g_{p}(t), g_{p, q}(t), g_{p, q, r}(t), \ldots$ are assumed to be the same for all genes in the RGC and since there are overlaps of cis elements among genes in this RGC, one can estimate these functions from an array of expression profiles $Y_{1}(t)$, $Y_{2}(t), \ldots, Y_{N}(t)$ of the genes in the RGC simultaneously, taking advantage of cross information enhancement. The RGCs for MFA2 and CLB2 are shown in Table 1 and Table 2 , respectively. 
Second, by integrating the dynamic equations of cis-regulatory circuits for $N$ genes in the RGC of the gene of interest, we obtain the following array of dynamic equations

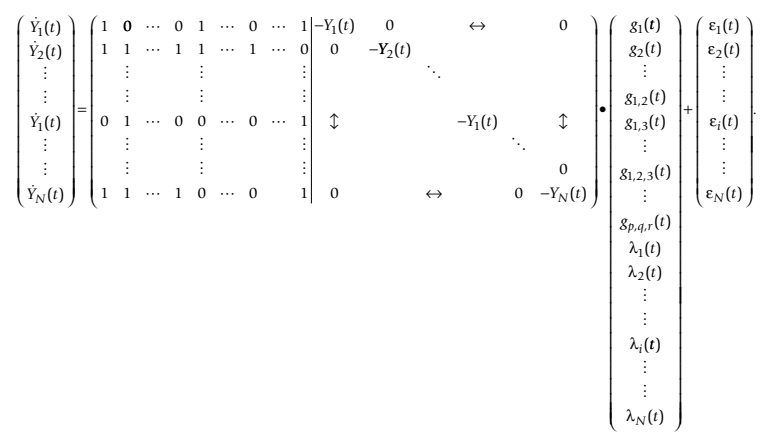

(12)

In the dynamic equations in (12), the regulatory functions $g_{p}(t), g_{p, q}(t)$ and $g_{p, q, r}(t)$ are shared by genes in the RGC. Therefore, the estimation of these functions from expression profiles $Y_{1}(t), Y_{2}(t), \ldots, Y_{N}(t)$ can use also information from other genes to enhance our ability to reconstruct the cis-regulatory circuit of the gene of interest.

Finally, since the number of functions $g_{p}(t), g_{p, q}(t), g_{p, q, r}(t)$, ... is finite, we can estimate these functions and the decay rates $\lambda_{1}(t), \lambda_{2}(t), \ldots, \lambda_{N}(t)$ if the number $N$ of dynamic equations in Equation (12) is large enough. Equation (12) can be rewritten in an algebraic form

$\tilde{\mathrm{X}}(t)=\tilde{\mathrm{A}}(t) \cdot \tilde{\mathrm{G}}(t)+\mathrm{E}(t)$,

where

$$
\tilde{\mathrm{X}}(t)=\left(\begin{array}{c}
\dot{Y}_{1}(t) \\
\dot{Y}_{2}(t) \\
\vdots \\
\vdots \\
\dot{Y}_{i}(t) \\
\vdots \\
\vdots \\
\dot{Y}_{N}(t)
\end{array}\right), \tilde{\mathrm{G}}(t)=\left(\begin{array}{c}
g_{1}(t) \\
g_{2}(t) \\
\vdots \\
g_{1,2}(t) \\
\vdots \\
g_{p, q, r}(t) \\
\lambda_{1}(t) \\
\lambda_{2}(t) \\
\vdots \\
\vdots \\
\lambda_{N}(t)
\end{array}\right), \mathrm{E}(t)=\left(\begin{array}{c}
\varepsilon_{1}(t) \\
\varepsilon_{2}(t) \\
\vdots \\
\vdots \\
\varepsilon_{i}(t) \\
\vdots \\
\vdots \\
\varepsilon_{N}(t)
\end{array}\right) .
$$

Then, we have the following dynamic equations for all time profiles

$$
\left(\begin{array}{c}
\tilde{\mathrm{X}}\left(t_{1}\right) \\
\tilde{\mathrm{X}}\left(t_{2}\right) \\
\vdots \\
\tilde{\mathrm{X}}\left(t_{m}\right)
\end{array}\right)=\left(\begin{array}{cccc}
\tilde{\mathrm{A}}\left(t_{1}\right) & 0 & \leftrightarrow & 0 \\
0 & \tilde{\mathrm{A}}\left(t_{2}\right) & & \uparrow \\
\uparrow & & \ddots & 0 \\
0 & \leftrightarrow & 0 & \tilde{\mathrm{A}}\left(t_{m}\right)
\end{array}\right) \cdot\left(\begin{array}{c}
\tilde{\mathrm{G}}\left(t_{1}\right) \\
\tilde{\mathrm{G}}\left(t_{2}\right) \\
\vdots \\
\tilde{\mathrm{G}}\left(t_{m}\right)
\end{array}\right)+\left(\begin{array}{c}
\mathrm{E}\left(t_{1}\right) \\
\mathrm{E}\left(t_{2}\right) \\
\vdots \\
\mathrm{E}\left(t_{m}\right)
\end{array}\right) .
$$

Let us denote the above equations in the following simple algebraic form

$\tilde{X}=\tilde{\Phi} \cdot \tilde{\Theta}+M$.

In Equation (15), $\tilde{\mathrm{X}}$ and $\tilde{\Phi}$ can be calculated from microarray data for the RGC of the gene of interest, which can then be used to estimate the vector $\tilde{\tilde{\Theta}}$ by the least squares method, leading to the following solution:

$\hat{\tilde{\Theta}}=\left(\tilde{\Phi}^{T} \tilde{\Phi}\right)^{-1} \tilde{\Phi} \tilde{X}$.

After $\hat{\tilde{\Theta}}$ is estimated from Equation (16), the regulatory functions $\tilde{\mathrm{G}}(t)$ in Equation (13) can be reconstructed for the genes in the RGC at every time point. However, in Equation (12), the degradation rate $\lambda(t)$ is a time-varying function and is affected by both the error terms and experimental data. Therefore, in order to reduce the influence on degradation rate, we simplify Equation (12) and average the negative gradients of $\lambda(t)$ to obtain the constant value $\hat{\lambda}$. Then the estimated $\hat{\lambda}$ is substituted into Equation (7) to re-identify the cis-regulatory functions to derive the final regulatory functions. Using this procedure, we can avoid the effects of the time-varying function $\lambda(t)$ on the identification process and reduce the influence by different experimental data. Hence, the degradation rate can be estimated. After the functions are estimated, they can be plugged into Equation (11) and then the reconstruction of the cis-regulatory circuit of the gene of interest is completed.

\section{Authors' contributions}

L.H. Lin carried out the model design and computation of this study, and drafted the manuscript. H.C. Lee participated in the design of the study and drafted the manuscript. W.H. Li amended and improved the design and the presentation of the study. B.S. Chen gave the topic and suggestions and was responsible for the entire study. All authors read and approved the final manuscript. 


\section{Additional material}

\section{Additional File 1}

The cell cycle genes and their connectivities to cis elements. 769 cell cycle genes defined by Spellman et al. [18] were selected from a total of 6178 genes in the data set. "1" denotes the connection of a cis element with a gene, while " 0 " means no connection. The main cis element data were compiled from the data set of Simon et al. 2001 [4] by choosing a P value (significance level) $\leq 0.0015$. Under this threshold, many interactions among cis elements for genes confirmed by the conventional data are included [35,36]. Additionally, we modified some cis element data, using well-known experimental information to correct false negatives [35,36]. Click here for file

[http://www.biomedcentral.com/content/supplementary/14712105-6-258-S1.xls]

\section{Acknowledgements}

We thank Ming-Che Shih, Geoff Morris, Jake Byrnes, Josh Rest and Ya-Wen Chang for helpful comments. This study was supported by an NSC grant NSC 91-2321-B-007-002, by Academia Sinica, Taiwan and by NIH grants.

\section{References}

I. Shea M, Ackers G: The OR control system of bacteriophage lambda. A physical-chemical model for gene-regulation. J Molec Biol 1985, |81:21।-230.

2. Mjolsness E, Sharp DH, Reinitz J: A connectionist model of development. J Theor Biol 1991, I 52:429-453.

3. lyer VR, Horak CE, Scafe CS, Botstein D, Snyder M, Brown PO: Genomic binding sites of the yeast cell-cycle transcription factors SBF and MBF. Nature 200I, 409:533-538.

4. Simon I, Barnett J, Hannett N, Harbison CT, Rinaldi NJ, Volkert TL Wyrick JJ, Zeitlinger J, Gifford DK, Jaakkola TS, Young RA: Serial regulation of transcriptional regulators in the yeast cell cycle. Cell 200I, 106:697-708.

5. Lee TI, Rinaldi NJ, Robert F, Odom DT, Bar-Joseph Z, Gerber GK, Hannett NM, Harbison CT, Thompson CM, Simon I, Zeitlinger J, Jennings EG, Murray HL, Gordon DB, Ren B, Wyrick JJ, Tagne JB, Volkert TL, Fraenkel E, Gifford DK, Young RA: Transcriptional regulatory networks in Saccharomyces cerevisiae. Science 2002, 298:799-804.

6. Gao F, Foat BC, Bussemaker HJ: Defining transcriptional networks through integrative modeling of mRNA expression and transcription factor binding data. BMC Bioinformatics 2004, 5:31

7. Wei $H$, Kaznessis $Y$ : Inferring gene regulatory relationships by combining target-target pattern recognition and regulatorspecific motif examination. Biotechnol Bioeng 2005, 89:53-77.

8. Haverty PM, Hansen U, Weng Z: Computational inference of transcriptional regulatory networks from expression profiling and transcription factor binding site identification. Nucleic Acids Res 2004, 32:179-188.

9. Kato M, Hata N, Banerjee N, Futcher B, Zhang MQ: Identifying combinatorial regulation of transcription factors and binding motifs. Genome Biol 2004, 5:R56.

10. Nachman I, Regev A, Friedman N: Inferring quantitative models of regulatory networks from expression data. Bioinformatics 2004, 20:1248-1256.

II. Wang W, Cherry JM, Nochomovitz Y, Jolly E, Botstein D, Li H: Inference of combinatorial regulation in yeast transcriptional networks: a case study of sporulation. Proc Natl Acad Sci USA 2005, 102: 1998-2003.

12. Yuh CH, Moore JG, Davidson EH: Quantitative functional interrelations within the cis-regulatory system of the $S$. purpuratus Endo I6 gene. Development 1996, I 22:4045-4056.

13. Yuh $\mathrm{CH}$, Bolouri H, Davidson EH: Genomic cis-regulatory logic: experimental and computational analysis of a sea urchin gene. Science 1998, 279: 1896-1902.
14. Yuh $\mathrm{CH}$, Bolouri $\mathrm{H}$, Davidson $\mathrm{EH}$ : Cis-regulatory logic in the endo 16 gene: switching from a specification to a differentiation mode of control. Development 200I, I 28:617-629.

I5. Banerjee N, Zhang MQ: Identifying cooperativity among transcription factors controlling the cell cycle in yeast. Nucleic Acids Res 2003, 3 I:7024-703I.

16. Das D, Banerjee N, Zhang MQ: Interacting models of cooperative gene regulation. Proc Natl Acad Sci USA 2004, I0I:16234-16239.

17. Chen HC, Lee HC, Lin TY, Li WH, Chen BS: Quantitative characterization of the transcriptional regulatory network in the yeast cell cycle. Bioinformatics 2004, 20:19|4-1927.

18. Spellman PT, Sherlock G, Zhang MQ, lyer VR, Anders K, Eisen MB, Brown $P Q$, Bostein $D$, Futcher $B$ : Comprehensive identification of cell cycle-regulated genes of the yeast Saccharomyces cererisiae by microarray hybridization. Mol Biol Cell 1998, 9:3273-3297.

19. Michaelis S, Herskowitz I: The a-factor pheromone of Saccharomyces cerevisiae is essential for mating. Mol Cell Biol 1988, 8:1309-1318

20. Wang Y, Liu CL, Storey JD, Tibshirani RJ, Herschlag D, Brown PO: Precision and functional specificity in mRNA decay. Proc Natl Acad Sci USA 2002, 99:5860-6865.

21. de Boor C: A Practical Guide to Splines New York: Springer-Verlag Press; 1978.

22. Bar-Joseph Z, Gerber G, Gifford DK, Jaakkola TS, Simon I: A new approach to analyzing gene expression time series data. In The Sixth Annual International Conference on Research in Computational Molecular Biology (RECOMB) Washington DC; 2002:39-48.

23. Maher M, Cong F, Kindelberger D, Nasmyth K, Dalton S: Cell cycleregulated transcription of the CLB2 gene is dependent on Mcml and a ternary complex factor. Mol Cell Biol 1995, I5:3|29-3I37.

24. Koranda M, Schleiffer A, Endler L, Ammerer G: Fork-head-like transcription factors recruit Ndd I to the chromatin of G2/Mspecific promoters. Nature 2000, 406:94-98.

25. Zhu G, Spellman PT, Volpe T, Brown PO, Botstein D, Davis TN Futcher $B$ : Two yeast forkhead genes regulate the cell cycle and pseudohyphal growth. Nature 2000, 406:90-94.

26. Kumar R, Reynolds DM, Shevchenko A, Goldstone SD, Dalton S: Forkhead transcription factors, Fkh Ip and Fkh2p, collaborate with $\mathrm{Mcm} / \mathrm{p}$ to control transcription required for $\mathrm{M}$ phase. Curr Biol 2000, 10:896-906.

27. Mclnerny CJ, Partridge JF, Mikesell GE, Creemer DP, Breeden LL: A novel $\mathrm{Mcm}$ I-dependent element in the SWI4, CLN3, CDC6, and CDC47 promoters activates M/GI-specific transcription. Genes Dev 1997, I I:1277-1288.

28. Dirick L, Moll T, Auer H, Nasmyth K: A central role for SWI6 in modulating cell cycle start-specific transcription in yeast. Nature 1992, 357:508-513.

29. Ho Y, Costanzo M, Moore L, Kobayashi R, Andrews BJ: Regulation of transcription at the Saccharomyces cerevisiae start transition by Stbl, a Swi6-binding protein. Mol Cell Biol 1999, 19:5267-5278.

30. Loy CJ, Lydall D, Surana U: NddI, a high-dosage suppressor of cdc28-IN, is essential for expression of a subset of late-S. phase-specific genes in Saccharomyces cerevisiae. Mol Cell Biol 1999, 19:3312-3327.

31. Lim FL, Hayes A, West AG, Pic-Taylor A, Darieva Z, Morgan BA Oliver SG, Sharrocks AD: Mcm Ip-induced DNA bending regulates the formation of ternary transcription factor complexes. Mol Cell Biol 2003, 23:450-46I.

32. Beer MA, Tavazoie S: Predicting gene expression from sequence. Cell 2004, I 17:185-198.

33. Yeast Cell Cycle Analysis Project [http://cellcycle-www.stanford.edu/]

34. McBride $\mathrm{HJ}, \mathrm{Yu} Y$, Stillman DJ: Distinct regions of the Swi5 and Ace2 transcription factors are required for specific gene activation. J Biol Chem 1999, 274:21 029-21036.

35. Cross FR, Hoek M, McKinney JD, Tinkelenberg AH: Role of Swi4 in cell cycle regulation of CLN2 expression. Mol Cell Biol 1994, 1 4:4779-4787.

36. Costanzo M, Schub O, Andrews B: GI Transcription factors are differentially regulated in Saccharomyces cerevisiae by the Swi6-binding protein Stb I. Mol Cell Biol 2003, 23:5064-5077. 